This item was submitted to Loughborough's Research Repository by the author.

Items in Figshare are protected by copyright, with all rights reserved, unless otherwise indicated.

\title{
Evidence that subclinical somatoform dissociation is not characterised by heightened awareness of proprioceptive signals
}

PLEASE CITE THE PUBLISHED VERSION

https://doi.org/10.1080/13546805.2016.1231112

\section{PUBLISHER}

(c) Taylor and Francis

\section{VERSION}

AM (Accepted Manuscript)

\section{PUBLISHER STATEMENT}

This work is made available according to the conditions of the Creative Commons Attribution-NonCommercialNoDerivatives 4.0 International (CC BY-NC-ND 4.0) licence. Full details of this licence are available at: https://creativecommons.org/licenses/by-nc-nd/4.0/

\section{LICENCE}

CC BY-NC-ND 4.0

\section{REPOSITORY RECORD}

Ratcliffe, Natasha, and Roger Newport. 2019. "Evidence That Subclinical Somatoform Dissociation Is Not Characterised by Heightened Awareness of Proprioceptive Signals". figshare.

https://hdl.handle.net/2134/28420. 
This is an Accepted Manuscript of an article published by Taylor \& Francis in Cognitive Neuropsychiatry on 23/09/16, available online:

http://www.tandfonline.com/10.1080/13546805.2016.1231112

Evidence that subclinical somatoform dissociation is not characterised by heightened awareness of proprioceptive signals

\section{Natasha Ratcliffe \& Roger Newport}

School of Psychology, University of Nottingham, Nottingham, NG7 2RD, UK.

Corresponding author:

\section{Natasha Ratcliffe}

School of Psychology, University of Nottingham

Nottingham, NG7 2RD, UK.

Email: 1pxnr2@nottingham.ac.uk

Tel: 01159515281

Roger Newport

School of Psychology, University of Nottingham

Nottingham, NG7 2RD, UK.

Email: roger.newport@nottingham.ac.uk

Tel: 01158467925

Funding: This work was supported by the BIAL Foundation under grant number 203/12.

Disclosure Statement: The authors have no financial relationships to disclose in relation to this work 


\begin{abstract}
Introduction: It has been suggested that abnormal perceptual processing and somatosensory amplification may be contributory factors to somatoform symptom reporting. A key source of somatosensory information is proprioception, yet the perception and integration of this sense has not been sufficiently investigated in those prone to somatoform disorders.

Methods: Sub-clinical groups of high- and low-scorers on the Somatoform Dissociation Questionnaire made judgements about the location of their unseen hand following congruent or incongruent visuo-proprioceptive feedback, which was manipulated using a MIRAGE mediated-reality system.

Results: No differences were found between groups, with both groups displaying normal proprioceptive accuracy under congruent conditions and equivalent visuo-proprioceptive integration under incongruent conditions.

Conclusions: The results suggest that amplification of, or abnormal weighting for, proprioceptive signals is not a contributing factor to somatoform symptom reporting.
\end{abstract}

Keywords: somatoform dissociation; medically unexplained symptoms; proprioception; sensory integration; MIRAGE

\title{
Introduction
}

Dissociative disorders are characterised by disruptions in the normal integration of mental processes, leading to a disconnection between thoughts, feelings, emotions, perceptions and memories. There are a number of recognised dissociative disorders that are characterised by different types of dissociative experience, including memory loss (dissociative amnesia), a sense of detachment from the self (depersonalisation) or shifts in identity (dissociative identity disorder). Other types of dissociative experience include 
autoscopic phenomena, such as the out of body experience (OBE). Nijenhuis, Spinhoven, Van Dyck, van der Hart, \& Vanderlinden (1996) coined the term 'somatoform dissociation' to describe a specific type of dissociative experience characterised by the presence of bodily symptoms for which no medical or organic cause can be determined. Also known as somatisation, presence of severe symptoms may lead to a diagnosis of somatic symptom disorder (SSD, DSM-5; formerly somatoform disorder in DSM-IV). While only a small percentage of the population meet this clinical diagnosis, the prevalence of somatisation in the general population is relatively high; so-called medically unexplained symptoms account for up to $30 \%$ of primary care consultations (Nimnuan, Hotopf, \& Wessely, 2001; Steinbrecher, Koerber, Frieser, \& Hiller, 2011) and up to $20 \%$ of secondary care cases (Reid, Wessely, Crayford, \& Hotopf, 2001). These figures highlight the importance of understanding the mechanisms contributing to medically unexplained symptoms in nonclinical populations as well as in clinical groups.

Early theories attempting to explain the occurrence of unexplained symptoms (referred to as "hysteria") emphasised the role of dissociation in symptom emergence and maintenance (e.g. Janet, 1907). Although later theories focussed on understanding unexplained symptoms in relation to normal physiological processes (Kirmayer \& Taillefer, 1997; Rief \& Barsky, 2005), current theoretical perspectives offer a more integrated approach, incorporating elements of dissociative accounts with other concepts (e.g. Brown, 2004). The link between unexplained symptoms and dissociation is supported by research showing that patients with dissociative disorders score highly on the Somatoform Dissociation Questionnaire (SDQ; developed by Nijenhuis et al., 1996), a measure of unexplained somatic symptoms, with the SDQ distinguishing between patients with dissociative disorders and those with other psychiatric disorders (Nijenhuis, 2010). SDQ scores have been found to correlate strongly with measures of dissociative experience across both clinical and nonclinical populations 
(Sar, Kundakci, Kiziltan, Bakin, \& Bozkurt, 2001), and out-of-body experiences reported in student populations are also associated with increased experience of somatoform dissociation (Irwin, 2000; Murray \& Fox, 2005) Taken together, these findings confirm the relevance of unexplained symptoms in a range of dissociative experiences.

A central feature of several models of medically unexplained symptoms is the suggestion that underlying abnormalities in perceptual processing play a significant role in the emergence and maintenance of symptoms. Barsky, Goodson, Lane, \& Cleary (1988) introduced the concept of somatosensory amplification, which describes the tendency to experience amplification of normal bodily sensations, such that benign or weak physiological sensations are perceived as intense, noxious and disturbing. Increased attention to these sensations subsequently leads to them being interpreted as abnormal and a sign of disease. Barsky (1992) suggests that this process of body hyper vigilance, attentional focus and misattribution may serve as a mechanism contributing to somatisation. Several studies have found a link between somatosensory amplification and somatic symptom reporting (Aronson, Barrett, \& Quigley, 2001; Duddu, Chaturvedi, \& Isaac, 2003; Muramatsu et al., 2002), although the nature of the relationship is unclear since both somatosensory amplification and somatisation are closely related to other factors such as anxiety and depression (Duddu, Isaac, \& Chaturvedi, 2006). Rief \& Barsky (2005) extend the suggested role of perceptual amplification in their signal-filtering model, suggesting that misperceptions may arise not only from amplification but also due to reduced filtering of sensory signals. This would allow otherwise unnoticed signals to reach conscious awareness, resulting in increased perception of sensory signals that may then be misattributed as illness. While these types of theories suggest that people experiencing somatoform symptoms may experience increased body awareness and stronger perceptions of internal signals, other models propose that perception is more strongly influenced by subjective representations, which may actually render it less 
accurate. Brown $(2004,2006)$ suggests that medically unexplained symptoms are brought about when representations are inappropriately selected during automatic attentional processes at the level of the primary attentional system. Since selection made at this level is automatically triggered, the inappropriate selection does not reach awareness, therefore the misconception is experienced as subjectively real despite sensory signals being inconsistent with these representations. This overreliance on "top-down" factors leads to perceptions that are less in line with reality.

Several studies have sought to clarify the nature of any perceptual abnormalities in those experiencing medically unexplained symptoms, and whether or not the perception of bodily signals is more or less accurate. Both the interoceptive and proprioceptive systems play an important role in body awareness. The interoceptive sense is responsible for monitoring the physiological state of the body (Craig, 2003), encompassing information from a number of internal systems such as the digestive, respiratory and cardiac systems. This is distinguishable from proprioception, the sense of the body's position and movement in space, which arises from sensory receptors in the muscles, joints and skin (Proske \& Gandevia, 2012). Paradigms investigating both interoceptive and proprioceptive awareness have sought to offer some insight into how these sensory signals are processed in those experiencing medically unexplained symptoms and somatoform disorders. Using an EMG-biofeedback task to investigate proprioceptive abilities, Scholz, Ott, \& Sarnoch (2001) found that patients with somatoform disorder showed more precise proprioception for muscle tension than a control group, lending support to the suggestion of heightened body awareness in somatoform disorders. However there were no differences in subjective ratings of perceived intensity of proprioception between the two groups, as the amplification account would predict. Findings from other studies have suggested that bodily perceptions are less accurate in those experiencing somatic symptoms. Bogaerts et al. $(2008,2010)$ used the Rebreathing 
Test to induce mild respiratory symptoms in participants, and compared subjective ratings of symptom experience with physiological referents. In both clinical and non-clinical samples, the correlation between subjective ratings and physiological measures was lower in those experiencing medically unexplained symptoms, indicating reduced interoceptive accuracy. Schaefer, Egloff, \& Witthöft (2012) also found that higher symptom reports were associated with reduced interoceptive accuracy, assessed using heartbeat perception tasks. Although taken together these findings provide a somewhat mixed picture of the way in which bodily signals are perceived and processed in those with medically unexplained symptoms, a common theme across findings is that perception is distorted in some way. The exact nature and direction of distortions may depend on the manner in which constructs such as bodily awareness, perceptual amplification and interoceptive accuracy are conceptualised. Whilst terms are often used interchangeably, it is possible that they represent distinct processes that all contribute to a wider multi-dimensional concept (Mehling et al., 2009).

Further support for the suggestion that abnormalities in processing of body related perceptual information contribute to the mechanisms underlying medically unexplained symptoms is provided by studies investigating how information from multiple sensory sources is processed. Brown, Brunt, Poliakoff, \& Lloyd (2010) found that high symptom reporters were more likely to experience illusory touch in the presence of a non-informative visual stimulus on the somatic signal detection task (SSDT), suggesting that medically unexplained symptoms are related to distortions in perceptual processing. The same group also report that high symptom reporters are less susceptible to the rubber hand illusion (Miles, Poliakoff, \& Brown, 2011), a well-established body illusion whereby synchronous visuo-tactile stimulation of a rubber hand and the participants own, unseen, hand leads to embodiment of the rubber limb. Subjective reports revealed that high symptom reporters had a decreased illusion experience compared to the low symptom group and, furthermore, their 
estimates of hand location (an objective measure of illusion susceptibility) displayed less drift towards the rubber hand. Since the illusion relies on the integration of sensory information from the visual, tactile and proprioceptive domains, altered experience of the illusion suggests the processing of sensory information in high symptom reporters may be different. For example, perceptual experience may be more driven by one sensory modality over another. The authors (Miles et al., 2011) suggest that bodily experience in high symptom reporters is more driven by "top-down" knowledge, whereby perceptions are more in line with cognitive representations. In the rubber hand illusion, this leads to a decrease in susceptibility due to the incompatibility of the rubber hand with existing representations of the body.

An alternative explanation for these findings is that perceptual experience is actually more driven by "bottom-up" sensory information in high symptom reporters, specifically proprioception. If, as some of the preceding literature suggests, internal signals are more accurately perceived in this group, proprioceptive information may be more strongly weighted in comparison to other sensory modalities. During the rubber hand illusion, in which there is a discrepancy between visuo-tactile and proprioceptive information, an increased reliance on proprioception could lead to a more stable and accurate sense of body position and, subsequently, reduced illusory experience. Whilst the paradigm used by Scholz et al. (2001) was designed to investigate proprioceptive muscle accuracy, no studies have investigated specifically how proprioceptive information is integrated in those susceptible to somatoform dissociation. Therefore the aim of the current experiment was to test the hypothesis that people scoring highly on the Somatoform Dissociation Questionnaire would weight proprioceptive information more strongly under conditions of multisensory conflict. The MIRAGE system used in the current experiment provides a novel way to investigate the way in which multisensory information is processed in this group. The system enables the 
experience of the self to be altered by changing sensory information relating to the participant's own hand, as oppose to a fake or virtual hand (e.g. Miles et al, 2011). This set up provides insight into how incongruent sensory information about one's own body is incorporated into the body representation. Even when sensory input clearly violates existing "top-down" knowledge about the body, manipulations can lead to significant changes in the way the body feels, which can have significant implications for many aspects of body perception, for example, the experience of pain (Preston \& Newport, 2011). By manipulating sensory information about one's own body, radical misperceptions can be induced, such as feeling as though one hand has completely disappeared (Newport \& Gilpin, 2011). Such paradigms allow investigation into the weighting placed on different sensory modalities during multisensory integration. For example, Bellan et al. (2015) used the MIRAGE system to induce a discrepancy between visual and proprioceptive information to investigate how these inputs are integrated across time under conditions of sensory conflict. They observed that whilst initially vision is weighted more strongly than proprioception, over time, as the visual trace of the hand decays, the weighting given to proprioceptive information gradually increases. The current study used a paradigm adapted from Bellan et al. (2015) to investigate how visual and proprioceptive inputs are weighted (following the introduction of sensory conflict) in people reporting higher levels of somatoform dissociation. Participants completed a hand localisation task following the introduction of a covert discrepancy between the visual and proprioceptive location of the hand. Based on the findings of Scholz et al. (2001) and the apparently more stable sense of body position observed in the high symptom group in the Miles et al. (2011) study, it was hypothesised that high symptom reporters would be more accurate at locating the position of their hidden hand following manipulation. Since reliance on proprioceptive information has been shown to increase when visual information about hand position is degraded or occluded (Bellan et al., 2015; Mon-Williams, Wann, Jenkinson, 
\& Rushton, 1997; Welch \& Warren, 1980), we also investigated perceptual drift across time in low and high symptom groups to further clarify the proprioceptive abilities in those susceptible to medically unexplained symptoms.

\section{Methods}

\section{Participants}

Participants were recruited through posters and advertisements; the majority of those who took part were university students. Participants were invited to complete an online version of the Somatoform Dissociation Questionnaire (SDQ; Nijenhuis et al., 1996) which was used to assess experience of unexplained symptoms. Participants were asked to indicate the extent to which they have experienced each of 20 symptoms during the past year on a scale ranging from not at all (1) to extremely (5). For items given a rating of 2 or above, participants were asked to state whether or not a physician had identified the physical cause for the symptom, and if so, to give brief detail of this. If the participant detailed a physical cause that had been identified by a physician, the item was rescored as 1 . Where participants had answered "yes" to the identification of a physical cause, but only provided a speculative description, the item was not rescored.

Total scores on the SDQ range from 20 to 100. Patients with somatoform disorders typically score over 30 (Chu, 2011). Since scores on this measure in the normal population are highly positively skewed, the SDQ was administered as a screening questionnaire with the aim of ensuring that a roughly equal number of low and high symptom reporters were invited to complete the experimental session. As participants were recruited from a non-clinical sample, the cut-off ranges for classification as low or high symptom reporters were $\leq 21$ and $\geq 26$ respectively. Scores of 30 or over have been taken to indicate high somatoform dissociation 
(Maaranen et al., 2004), however in experimental research a cut-off point of 28 has commonly been adopted to classify participants as high scorers (e.g. Brown et al., 2010; Miles et al., 2011). The cut-off for the high group in the current study was slightly lower than previous experimental studies have used. However, we observed significant positive skew in SDQ scores, with fewer than $15 \%$ of all participants completing the SDQ $(\mathrm{N}=80)$ scoring 28 or above. Furthermore, since the study aimed to capture somatoform dissociation in nonclinical populations, the decision was taken to adopt a revised cut-off point for the high group of 26 for the high group, which enabled elevated symptom scores representative of this population to be captured. Fifty-five participants scored within the cut-off ranges and were invited to complete the experimental session; 34 of whom took part. Three participants were excluded from the analysis; two due to the presence of outlier data in the incongruent condition ( $>2$ standard deviations below the mean), and one due to a substantially higher SDQ-score than the rest of the sample ( $>5$ standard deviations above the mean), This resulted in a sample size of 31 participants, split into a low-SDQ group $(N=17,8$ male $)$ and a highSDQ group ( $N=14,8$ male). Table 1 displays descriptive statistics for participants by group.

[Table 1 about here]

\section{Questionnaire measures}

Since perceptual amplification is thought to be relevant for experience of somatoform symptoms, participants completed the Somatosensory Amplification Scale (SSAS; Barsky et al., 1990), which measures the tendency to experience amplification of normal sensations. The State-trait anxiety inventory (STAI-T; Spielberger et al., 1983) was used to measure anxiety, which has been linked to both increased symptom reporting and somatosensory amplification (Duddu et al., 2006). 


\section{Apparatus}

The experiment was conducted using the MIRAGE system (Newport, Preston, Pearce, \& Holton, 2009) controlled using LabVIEW (National Instruments LabVIEW version 2010). MIRAGE is a mediated reality system that allows the participant to view a real-time video image of their own hands in the same apparent physical location as their real hands. A camera records a live video image (delay of $\sim 16 \mathrm{~ms}$ ) of the work surface, which is fed to a display screen and reflected to the participant by a mirror suspended equidistant between the display screen above and work surface below. The angle of the camera is such that the hands in the image are presented in the same spatial location as the user's actual hands, thus giving the participant the impression that he or she is viewing their hands directly. Custom software allows the size, appearance and location of the hand to be manipulated in various ways, resulting in incongruous sensory information from different modalities and/or conflict between sensory information and "top-down" knowledge. Previous experimental manipulations have involved creating the feeling that the participant has extra hands (Newport, Pearce, \& Preston, 2010), extended fingers (Preston \& Newport, 2011) and creating the illusion that the participant's hand has disappeared (Newport \& Gilpin, 2011).

\section{Procedure}

The participant was seated at the MIRAGE device with both hands placed on the work surface. A black cloth, covering both shoulders and arms, was loosely fastened round the participant's neck so that the angle and location at which the hands entered MIRAGE could not be seen. The experiment required the participant to judge the location of their seen or unseen hands following exposure to congruent or incongruent visuo-proprioceptive sensory 
information about the location of their hands. Judgements were made under four conditions: Congruent Seen (CS), Congruent Unseen Inner (CUI), Congruent Unseen Outer (CUO) and Incongruent Unseen (IU). In the congruent conditions, the seen location of both hands was congruent with the actual location of the hands. At the start of each condition, the participant watched as the experimenter positioned the participant's hands either side of the body midline. The distance between each index finger and the midline was $58.5 \mathrm{~mm}$ for conditions Congruent Seen and Congruent Unseen Inner and $153.5 \mathrm{~mm}$ in condition Congruent Unseen Outer. These two distances corresponded to the seen and actual location of the hand, respectively, in the Incongruent Unseen condition (see below), and were included to check for any potential differences in accuracy depending on hand eccentricity. In condition Congruent Seen, included to verify task understanding, the hands remained visible for the localisation judgements. In Congruent Unseen Inner and Congruent Unseen Outer, a blank (black) image replaced and covered the image of the hands before participants completed the judgements.

In the incongruent condition, the image of the hands was manipulated such that the seen location of the right hand was incongruent with its actual location (note: the seen and actual locations of the left hand remained congruent). This was achieved using an adaptation procedure similar to that used by Newport \& Gilpin (2011) and Bellan et al. (2015). Three thin blue bars were superimposed on the outer edges and centre of the viewed workspace, leaving the participant's hands visible in the spaces between (see Figure 1a). Over the course of 25 seconds the bars expanded, reducing the space in which the hands could be seen (Figure 1b). Keeping their hands and forearms elevated $\sim 5 \mathrm{~cm}$ above the work surface, participants were instructed to ensure that their hands did not touch the blue bars on either side and keep them visible within the space between as it reduced. During this procedure, the image of the right hand was manipulated to move slowly leftwards at a rate of $4.5 \mathrm{~mm} / \mathrm{s}$ meaning that, in 
order to keep the hand visible and in the same place visually, participants had to move their hand rightwards at the same rate (Figure 1c). This resulted in a discrepancy between the seen and actual location of the right hand, such that at the end of the procedure, the right hand was seen to be $11.25 \mathrm{~cm}$ to the left of its actual location. Throughout this time the image of the left hand slowly oscillated laterally, but the final seen location of the hand was congruent with its actual location. The movement of the right hand happens so slowly that it is unnoticeable; no participant has reported conscious awareness of the visuo-proprioceptive discrepancy in previous experiments (Bellan et al., 2015; Newport \& Gilpin, 2011). After the 25-second adaptation procedure, the experimenter guided the participant's hands to rest on the work surface. The hand image was then replaced by a blank image (as in Congruent Unseen Inner and Congruent Unseen Outer) before judgements were completed.

[Figure 1 about here]

Localisation judgements were completed immediately following the occlusion of the hand image in the unseen conditions (Congruent Unseen Inner, Congruent Unseen Outer and Incongruent Unseen; in Congruent Seen, judgements were made immediately after positioning of the hands). Participants were asked to say "stop" when a red arrow moving horizontally across the image was directly above where they felt their index finger to be, aiming for the middle of the index finger (see Figure 1d). The arrow travelled either from left to right or from right to left. The appearance of the arrow and the onset and offset of its movement were controlled by the experimenter, although the speed of its movement was automatic so that the arrow moved smoothly and at the same pace for all participants across all conditions. If immediately after saying "stop" the participant felt that the arrow had not stopped in the correct location, they could instruct the experimenter to manually adjust the 
position of the arrow backwards or forwards until it came to the desired location. The arrow disappeared promptly after the response was given and the experimenter manually recorded the $\mathrm{x}$-axis coordinate of the arrow location as the measure of perceived finger location. One set of judgements consisted of four trials - one trial for each travelling direction (leftright/right-left) for each finger (left/right) - and took approximately one minute to complete. The order of trials in each set was randomised. Before each trial, the experimenter specified which index finger the participant should judge and which direction the arrow would travel from. For conditions Congruent Seen, Congruent Unseen Inner and Congruent Unseen Outer, participants completed one set of judgements. In condition Incongruent Unseen, participants completed 7 sets of judgements separated by 10 -second intervals, across a time period totalling approximately 8 minutes. The hands remained hidden throughout all intervals and judgement trials. All participants completed the Congruent Seen condition first, to ensure that they understood the task, followed by the Congruent Unseen Inner, Congruent Unseen Outer and Incongruent Unseen conditions. In all conditions, the experimenter recorded the actual location of each index finger immediately before the judgements began. Participants were instructed to keep their hands still throughout the judgements and were specifically asked not to move their index finger to prevent any possibility of proprioceptive updating.

\section{Analysis}

The $\mathrm{x}$-axis coordinate of the actual hand location and each judgement were recorded in pixels and converted into $\mathrm{cm}(1 \mathrm{pixel}=0.73 \mathrm{~mm})$. For each set of judgements in each condition, a mean localisation estimate was calculated for each hand (averaged across arrow direction). The actual hand location was subtracted from the estimate to provide a measure of localisation error. Right hand values were multiplied by minus one so that, rather than reflecting error to the left or right of actual hand location, the localisation error scale reflected 
error towards or away from the body midline. Positive error values indicate estimates towards the body midline, while negative error values indicate estimates away from the body midline. Thus in condition Incongruent Unseen, since the seen location of the hand is closer to the body midline than the actual location of the hand, positive estimates indicate a shift in perceived hand location towards the location of the seen hand.

To investigate accuracy of hand localisation between the groups in the congruent conditions, a 3-way mixed ANOVA $(3 \times 2 \times 2)$ was conducted with the factors condition (Congruent Seen, Congruent Unseen Inner, Congruent Unseen Outer), hand (left, right) and SDQ group (low, high). A linear mixed model was used to investigate accuracy across time in condition Incongruent Unseen. Mixed models provide an alternative to the traditional ANOVA approach for analysing repeated measures data. ANOVA requires data from different conditions to be independent. However, collecting multiple measurements per participant across different conditions violates this assumption, since it introduces potential correlation between data points. Repeated measures ANOVA deals with this by assuming that the variance and covariance is the same across all repeated measures. Alternatively, mixed models control for dependence through the addition of random factors. Random factors give structure to the error term of the model and enable individual differences to be controlled for (Winter, 2013). Contrary to ANOVA, mixed models yield accurate results for unbalanced designs, and furthermore are better able to deal with missing values in the data. They also allow time to be modelled as a continuous variable, reducing the number of post- hoc comparisons that are required compared to if time is treated as categorical as is the typical approach in ANOVA.

To investigate whether incongruent visuo-proprioceptive information for the right hand affected localisation error, and whether or not this changed over time in each group, a linear mixed model was constructed using the mixed function in the R package afex (Singmann, 
Bolker, \& Westfall, 2015). Hand, Time (8 minute period from first judgement to seventh judgement) and SDQ group were entered as fixed factors and participant was entered as a random factor. The model included a random intercept for participant and random slopes for Hand and Time. Furthermore, because of the relatively small sample size in each SDQ group, a Bayesian analysis was conducted using the R package BayesFactor (Morey \& Rouder, 2015) in order to quantify the evidence for the null hypothesis compared to the alternative hypothesis $\left(\mathrm{BF}_{01}\right)$ for statistical tests involving the factor SDQ group. $\mathrm{A} \mathrm{BF}_{01}$ value of 10 indicates that the observed data were 10 times more likely to occur under the null hypothesis than the alternative.

\section{Results}

\section{ANOVA for congruent conditions}

Although there were an unbalanced number of participants in each SDQ group, neither Box's M test of equality of covariance matrices nor Levene's test of equality of error variances were significant at the $p=.05$ level. The data met all other assumptions of repeated measures ANOVA. Overall, accuracy was very high in all congruent conditions. Table 2 displays the mean localisation error for each hand in each condition for each SDQ group. Localisation error did not differ across conditions, $F(2,58)=1.80, p=.174$; hands, $F(1,29)=.716, p=$ .404 , or between SDQ groups, $F(1,29)=.736, p=.398$, and there were no interactions between any of the three factors (all $p>.1$ ). As predicted, both groups remained highly accurate in locating both hands when vision of the hands was removed, and, importantly, distance from the body midline (Congruent Unseen Inner vs. Congruent Unseen Outer) did not affect localisation error. 
[Table 2 about here]

\section{Linear mixed model for condition Incongruent Unseen}

Mean localisation errors in the Incongruent Unseen condition are shown in Figure 2. The presence of incongruent visuo-proprioceptive information for the right hand had a significant effect on localisation error, as evidenced by the main effect of hand, $F(1,40.12)=251.51, p$ $<.001)$. Error values were significantly higher for the right hand compared to the left hand, with error in the direction of the seen location of the right hand. Furthermore, there was a significant effect of time, $F(1,29.00)=6.98, p=.01$, although the interaction between hand and time was not significant, $F(1,335.42)=1.38, p=.24$.

Crucially, the results also revealed that SDQ group did not have a significant effect on localisation error. The main effect of SDQ group was not significant, $F(1,29.01)=.07, p=$ $.79, \mathrm{BF}_{01}=2.16 \pm 13.24 \%$, nor were the two-way interactions between SDQ group and hand, $F(1,40.12)=1.40, p=.24, \mathrm{BF}_{01}=0.88 \pm 14.07 \%$, and SDQ group and time, $F(1,29.00)=$ $.08, p=.78, \mathrm{BF}_{01}=10.63 \pm 17.25 \%$. Furthermore, the three-way interaction between SDQ group, hand and time was not significant, $F(1,335.42)=.80, p=.37, \mathrm{BF}_{01}=16.88 \pm 22.24 \%$.

[Figure 2 about here]

\section{Questionnaire Scores}

Independent samples t-tests were conducted to establish whether somatosensory amplification (SSAS) or trait anxiety (STAI-T) scores differed between SDQ groups. The high-SDQ group had a significantly higher mean SSAS score than the low-SDQ group (32.15 vs. 27.71), $t(28)=-2.11, p=.045$. STAI-T scores did not differ between groups, $t(29)$ $=-.40, p=.69$. 
A correlation analysis revealed no relationship between SSAS scores and mean localisation error (across all times) in the incongruent condition, $r(29)=-.05, p=.77$, therefore the analysis was not re-run with SSAS as a covariate.

\section{Discussion}

To examine the hypothesis that there is a relationship between heightened body awareness and somatoform dissociation in a sub-clinical population, proprioceptive localisation and visuo-proprioceptive integration were assessed through hand localisation judgements under congruent and incongruent sensory conditions. Similar patterns of responding were observed in both high- and low- scoring SDQ groups across all conditions. In the congruent conditions, for which visual information regarding the positions of the hands corresponded to their actual locations, all participants gave accurate estimates of hand position, even when the hands were hidden from view. When the visual information regarding the position of the right hand was displaced, rendering visual and proprioceptive information incongruent, localisation accuracy was significantly worse. Both groups initially perceived the right hand to be approximately two-thirds towards the visual representation of the hand (away from the real hand location). Furthermore, in the continued absence of visual information, it might be expected that the felt position of the hand would drift back towards the real location of the hand more rapidly in the high-SDQ group if that group had greater awareness of bodily sensations. However, for both the high- and low- SDQ groups, hand estimates remained consistently inaccurate, drifting only slightly towards the real position of the hand and consistently being mis-localised as closer to the visual hand than to the real hand for 8 minutes. The results therefore do not support the notion that those experiencing somatoform dissociative symptoms have increased body awareness or heightened sensitivity 
to body-related stimuli in terms of proprioceptive position sense. Indeed, the high-scoring group demonstrated normal proprioception, performing in a similar fashion to the lowscoring group.

This observation dismisses two potential alternative explanations for the findings of Miles et al. (2011) that previously remained unexplored. In that study, participants reporting higher levels of somatoform dissociation (SDQ $\geq 28$ ) were found to be less susceptible to the rubber hand illusion, identifying less with the rubber hand and showing less proprioceptive drift than the low-scoring group. The authors suggested that this finding might be a result of perceptions being more driven by top-down factors, such as limb appearance, in the highSDQ group. As the rubber hand, while relatively life-like, was obviously not a real arm, people more in tune with top-down cognitive knowledge would be less likely to embody the limb, while those driven more by bottom-up sensory information (congruent vision and touch) would embody the limb more. However, this finding could be accounted for by two alternative explanations. On one hand, it may be that the high-SDQ group had a greater awareness of the real position of their hidden limb, through increased body awareness or more precise proprioception (Scholz et al., 2001). In the rubber hand illusion, increased proprioceptive awareness could result in this sense being weighted more strongly than visual and tactile inputs during multisensory integration. In this case, the visuotactile synchrony observed at the rubber hand may be insufficient to override the felt position of the limb, resulting in less proprioceptive drift towards the fake limb as well as a greater subjective awareness that the fake limb could not be theirs due to its incorrect location. Thus, rather than perception in the high-SDQ group being driven by top-down influences, reduced illusion experience in this group may actually reflect a tendency for perception to be driven by a specific mode of "bottom-up" sensory information, namely proprioception. On the other hand, it may be the case that those in the high-SDQ group are poorer at integrating 
multisensory information. The rubber hand illusion depends on the successful integration of visual, tactile and proprioceptive signals (Makin, Holmes, \& Ehrsson, 2008) and a failure to bind together the seen and felt position of the hand (which are in different locations in the standard illusion) would reduce or eradicate the illusion. Somatoform dissociation implies a lack of integration for, or a disconnection between the processing of, cognitive and perceptual information related to the body (Nijenhuis, 2000) which, potentially, could extend to the integration and interpretation of basic multisensory signals. Therefore, the reduced illusion experience observed in those reporting a higher number of somatoform symptoms in the Miles et al. (2011) study may therefore indicate a failure to integrate multisensory information efficiently.

However, by demonstrating comparable performance across both the low- and highSDQ groups, the results of the current study appear to rule out both of these alternative explanations. In the incongruent condition, the high-SDQ group did not show an initial increase in accuracy in localising the right hand, nor did their estimates become any more accurate over time, compared to the low-SDQ group, providing no evidence that high-SDQ scorers have a heightened sense of limb position via proprioception under conditions of visuo-proprioceptive conflict. In addition to having normal proprioception, the high-SDQ group demonstrate the ability to integrate visual and proprioceptive information, evidenced by the fact that their localisation judgements of the right hand in the incongruent condition lie between the seen (visual) and actual (proprioceptive) location of the hand. Furthermore, the weighting given to visual and proprioceptive information is comparable across both groups. Although the group mean differences point towards a slight tendency for the high-SDQ group to weight visual information more strongly than proprioceptive information, this difference was not statistically significant, and by the end of the judgement period in the incongruent condition both the low- and high-SDQ groups gave approximately equal weighting to visual 
and proprioceptive information (group mean weighting towards visual information at final judgement: low $=60.38 \%$, high $=63.08 \%$ ).

Taken in tandem, these two observations are a clear sign that, in terms of proprioception at least, the sensory processing of those who might be susceptible to somatoform disorders or medically unexplained symptoms is not characterised by increased reliance on "bottom-up" sensory information arising from the body, nor a failure to integrate multisensory inputs. Instead, a greater weighting for top-down information seems to be the more plausible explanation for the Miles et al. (2011) study, which is in line with the model proposed by Brown $(2004,2006)$ suggesting misperceptions in those experiencing unexplained symptoms arise due to overreliance on top-down representations.

The purpose of the current study was to investigate the existence of abnormalities in perceptual processing that have been suggested to be a potential mechanism through which medically unexplained symptoms emerge and are maintained (Rief \& Barsky, 2005). The tendency to experience amplification of normal bodily sensations, interpreting innocuous sensory input as abnormal or harmful, along with abnormal attentional focus on these sensations may contribute to somatisation and the perception of disease in both clinical and subclinical populations (Barsky \& Borus, 1999; Houtveen, Rietveld, \& De Geus, 2003). Consistent with this hypothesis, the high-SDQ group reported significantly more somatosensory amplification than the low-SDQ group, which is in line with observations in previous studies (Brown, Poliakoff, \& Kirkman, 2007; Miles et al., 2011). However, somatosensory amplification did not correlate with localisation error in the incongruent condition, suggesting that general perceptual amplification does not imply heightened awareness of proprioceptive signals. Nonetheless, it remains possible that this could contribute to maintenance of somatoform symptoms in clinical groups; it may be that abnormalities in the processing of sensory signals are a consequence, rather than a cause, of 
persistent symptom experience, perhaps driven by other factors associated with increased perceptual amplification such as anxiety or depression (Duddu et al., 2006). Future research should focus on understanding the relationship between sensory integration, perceptual amplifcation and somatoform dissociation, in order to clairfy the processes underlying persistent symptom experience. This may also shed light on important differences between subclincal and clincal experience of somatoform dissociation.

Since the sample size in each of the SDQ groups was relatively small, a Bayes factor analysis was conducted to further investigate how strongly the data supported each null model over the alternative. For models involving the factor Time the analyses showed strong support for the null model (no interaction with SDQ group), indicating that the null findings were not due to insufficient evidence. The Bayes factors for the main effect of SDQ group and hand by SDQ group interaction were small, suggesting that the data do not strongly support either the null or alternative hypothesis. This indicates that a larger sample size is required to quantify these effects, and as such the present findings should be interpretted with some caution. However, when considering this in relation to the group means presented in Figure 2, it appears likely that any change in the effects due to increased sample size would likely be in the opposite direction to that predicted; that is, the high SDQ group may show a stonger weighting for vision. Although speculative, such a finding would still support the conclusion of the current study that those reporting more somatoform symptoms do not show heightened awareness for proprioception. However, it would point towards abrnomal multisensory integration of vision and proprioception in this group, therefore further research with a larger sample is necessary to clarify the effects.

While the current results do not point to an over-reliance or higher-perception of body position sense in people scoring highly on somatoform dissociation, they do not rule out the possibility that normal proprioceptive signals are subjectively perceived in an aberrant 
manner in these individuals. Although perception and integration of basic sensory signals (vision and proprioception) appears to be intact in those reporting somatoform dissociation, integration failures may occur further along in the processing stream, when sensory information needs to be combined with higher order representations. Indeed, this in line with proposed accounts of dissociative experiences such as the out-of-body experience, which are thought to arise due to a disintegration of low-level multisensory inputs and high-level selfprocessing at the temporo-parietal junction (Blanke \& Arzy, 2005), and also fits with suggestions that dissociative experiences in depersonalisation arise due to impairment in the integration of perception with emotional experience (Sierra \& David, 2011). Whilst these accounts focus on higher-order disruptions in multisensory integration, importantly, there actually appears to be little research examining how exteroceptive and proprioceptive signals are perceived and integrated in people experiencing anomalous/dissociative experiences. A study by Braithwaite, Broglia, \& Watson (2014) investigated how visual, proprioceptive and tactile signals are integrated during the rubber hand illusion in people reporting anomalous body experiences (ABEs), finding evidence for differences in temporal integration of multisensory signals, and psychophysiological components of the illusion, in those reporting more ABEs. As previously discussed, Miles et al. (2011) investigate this in those reporting somatoform dissociation, and the current study also sheds light on the way in which multisensory information is processed in this group. However, further research should look at similar processes in groups displaying other types of dissociation, such as the out-of-body experience, in order to fully understand the nature of the disruptions in multisensory integration that appear to be characteristic of dissociative experiences.

Finally, it is worth noting for how long localisation of the hidden hand remained inaccurate. It was expected that the felt location of the hand would drift back to the location of the real hand as proprioceptive position sense was updated in the absence of vision. 
However, the spatial representation of the unseen hand remained remarkably stable, staying more than $6 \mathrm{~cm}$ to the left of the real hand and drifting only around $0.5 \mathrm{~cm}$ over the final 7 minutes of visual occlusion. This is interesting because it shows that while body representation is very malleable when provided with new sensory information, it is also remarkably stable when not. Such discrepancy might help to explain some of the aberrant experiences observed in clinical populations such as complex regional pain syndrome (CRPS) and following stoke. If rapid cortical reorganisation of body representation, as a result of trauma to the brain or body, is followed by slow, incomplete or incorrect recalibration, due to maladaptive processes in CRPS or sensory loss/brain damage in stroke, then the stability of those aberrant representations might persist longer than would be expected such that limbs may become subjectively lost or alien.

The amount of drift observed for the right hand in the incongruent condition in the current study is less than the amount reported by Bellan et al. (2015), who observed drift of approximately $2.5 \mathrm{~cm}$ over the course of three minutes, compared to just under $2 \mathrm{~cm}$ over the course of 8 minutes in the current study. It seems logical to expect more, or at least as much, drift across an 8-minute period compared to a 3-minute period. One difference between the two studies that may account for this finding is that in the current study, participants made estimates of both the left hand and right hand location, with left hand judgements potentially providing an anchor for right hand judgements. Alternatively, it may be that judgements in the Bellan study would have stabilised had they been taken over a longer time period.

In summary, this experiment investigated the proprioceptive position sense and visuoproprioceptive integration in a group scoring highly on the somatoform dissociation questionnaire, observing no differences in proprioceptive accuracy or visuo-proprioceptive integration, suggesting that proprioception and sensory integration in high-scorers is normal. 
The study, therefore, does not support the notion that somatoform dissociation is underpinned by the amplification of proprioceptive signals. 


\section{References}

Aronson, K. R., Barrett, L. F., \& Quigley, K. S. (2001). Feeling your body or feeling badly Evidence for the limited validity of the Somatosensory Amplification Scale as an index of somatic sensitivity. Journal of psychosomatic research.

Barsky, A. J. (1992). Amplification, somatization, and the somatoform disorders. Psychosomatics, 33(1), 28-34. doi:10.1016/S0033-3182(92)72018-0

Barsky, A. J., \& Borus, J. . (1999). Functional somatic syndromes. Annals of Internal Medicine, 130(11), 910-921. doi:10.1016/S0140-6736(05)76818-5

Barsky, A. J., Goodson, J. ., Lane, R. ., \& Cleary, P. . (1988). The amplification of somatic symptoms. Psychosomatic Medicine, 50(5), 510-519. doi:10.1097/00006842198809000-00007

Barsky, A. J., Wyshak, G., \& Klerman, G. L. (1990). The somatosensory amplification scale and its relationship to hypochondriasis. Journal of Psychiatric Research, 24(4), 323334.

Bellan, V., Gilpin, H. R., Stanton, T. R., Newport, R., Gallace, A., \& Moseley, G. L. (2015). Untangling visual and proprioceptive contributions to hand localisation over time. Experimental Brain Research. doi:10.1007/s00221-015-4242-8

Blanke, O., \& Arzy, S. (2005). The Out-of-Body Experience: Disturbed Self-Processing at the Temporo-Parietal Junction. The Neuroscientist, 11(1), 16-24. doi:10.1177/1073858404270885

Bogaerts, K., Millen, A., Li, W., De Peuter, S., Van Diest, I., Vlemincx, E., ... Van den Bergh, O. (2008). High symptom reporters are less interoceptively accurate in a symptom-related context. Journal of Psychosomatic Research, 65(5), 417-24. doi:10.1016/j.jpsychores.2008.03.019

Bogaerts, K., Van Eylen, L., Li, W., Bresseleers, J., Van Diest, I., De Peuter, S., ... Van den 
Bergh, O. (2010). Distorted Symptom Perception in Patients With Medically Unexplained Symptoms. Journal of Abnormal Psychology, 119(1), 226-234.

Braithwaite, J. J., Broglia, E., \& Watson, D. G. (2014). Autonomic emotional responses to the induction of the rubber-hand illusion in those that report anomalous bodily experiences: evidence for specific psychophysiological components associated with illusory body representations. J Exp Psychol Hum Percept Perform, 40(3), 1131-1145. doi:10.1037/a0036077

Brown, R. J. (2004). Psychological mechanisms of medically unexplained symptoms: an integrative conceptual model. Psychological Bulletin, 130(5), 793-812. doi:10.1037/0033-2909.130.5.793

Brown, R. J. (2006). Medically unexplained symptoms: a new model. Psychiatry, 5(2), 4347. doi:10.1383/psyt.2006.5.2.43

Brown, R. J., Brunt, N., Poliakoff, E., \& Lloyd, D. M. (2010). Illusory touch and tactile perception in somatoform dissociators. Journal of Psychosomatic Research, 69(3), 2418. doi:10.1016/j.jpsychores.2009.11.010

Brown, R. J., Poliakoff, E., \& Kirkman, M. a. (2007). Somatoform dissociation and somatosensory amplification are differentially associated with attention to the tactile modality following exposure to body-related stimuli. Journal of Psychosomatic Research, 62(2), 159-65. doi:10.1016/j.jpsychores.2006.08.008

Chu, J. A. (2011). Appendix 2 : The Somatoform Dissociation Appendix : Rebuilding Shattered Lives, 282-286.

Craig, a. D. (2003). Interoception: The sense of the physiological condition of the body. Current Opinion in Neurobiology, 13(4), 500-505. doi:10.1016/S0959-4388(03)000904

Duddu, V., Chaturvedi, S. K., \& Isaac, M. K. (2003). Amplification and attribution styles in 
somatoform and depressive disorders - A study from Bangalore, India.

Psychopathology, 36, 98-103. doi:10.1159/000070365

Duddu, V., Isaac, M. ., \& Chaturvedi, S. . (2006). Somatization, somatosensory

amplification, attribution styles and illness behaviour: a review. International Review of Psychiatry (Abingdon, England), 18(1), 25-33. doi:10.1080/09540260500466790

Houtveen, J. H., Rietveld, S., \& De Geus, E. J. C. (2003). Exaggerated perception of normal physiological responses to stress and hypercapnia in young women with numerous functional somatic symptoms. Journal of Psychosomatic Research, 55, 481-490. doi:10.1016/S0022-3999(03)00011-4

Irwin, H. J. (2000). The Disembodied Self: An Empirical Study of Dissociation and the Outof-Body Experience. The Journal of Parapsychology, 64(3), 261.

Janet, P. (1907). The major symptoms of hysteria. New York; London: Macmillan.

Kirmayer, L. J., \& Taillefer, S. (1997). Somatoform disorders. In Adult psychopathology and diagnosis (pp. 333-383).

Maaranen, P., Tanskanen, A., Haatainen, K., Koivumaa-Honkanen, H., Hintikka, J., \& Viinamaki, H. (2004). Somatoform dissociation and adverse childhood experiences in the general population. Journal of Nervous \& Mental Disease, 192(5), 337-342. doi:10.1097/01.nmd.0000126700.41047.83

Makin, T. R., Holmes, N. P., \& Ehrsson, H. H. (2008). On the other hand: dummy hands and peripersonal space. Behavioural Brain Research, 191(1), 1-10. doi:10.1016/j.bbr.2008.02.041

Mehling, W. E., Gopisetty, V., Daubenmier, J., Price, C. J., Hecht, F. M., \& Stewart, A. (2009). Body awareness: construct and self-report measures. PloS One, 4(5), e5614. doi:10.1371/journal.pone.0005614

Miles, E., Poliakoff, E., \& Brown, R. J. (2011). Medically unexplained symptom reports are 
associated with a decreased response to the rubber hand illusion. Journal of Psychosomatic Research, 71(4), 240-4. doi:10.1016/j.jpsychores.2011.04.002

Mon-Williams, M., Wann, J. P., Jenkinson, M., \& Rushton, K. (1997). Synaesthesia in the normal limb. Proceedings. Biological Sciences / The Royal Society, 264(1384), 10071010. doi:10.1098/rspb.1997.0139

Morey, R. ., \& Rouder, J. . (2015). BayesFactor: Computation of Bayes Factors for Common Designs. R package version 0.9.11-1.

Muramatsu, K., Miyaoka, H., Muramatsu, Y., Fuse, K., Yoshimine, F., Kamijima, K., ... Sakurai, K. (2002). The amplification of somatic symptoms in upper respiratory tract infections. General Hospital Psychiatry, 24(3), 172-5. doi:10.1016/S01638343(02)00177-9

Murray, C. D., \& Fox, J. (2005). Dissociational body experiences: differences between respondents with and without prior out-of-body-experiences. British Journal of Psychology (London, England: 1953), 96(Pt 4), 441-56. doi:10.1348/000712605X49169

Newport, R., \& Gilpin, H. R. (2011). Multisensory disintegration and the disappearing hand trick. Current Biology : CB, 21(19), R804-5. doi:10.1016/j.cub.2011.08.044

Newport, R., Pearce, R., \& Preston, C. (2010). Fake hands in action: embodiment and control of supernumerary limbs. Experimental Brain Research., 204(3), 385-95. doi:10.1007/s00221-009-2104-y

Newport, R., Preston, C., Pearce, R., \& Holton, R. (2009). Eye rotation does not contribute to shifts in subjective straight ahead: Implications for prism adaptation and neglect. Neuropsychologia, 47(8-9), 2008-2012. doi:10.1016/j.neuropsychologia.2009.02.017

Nijenhuis, E. R. S. (2000). Somatoform Dissociation : Major Symptoms of Dissociative Disorders. Journal of Trauma and Dissociation, 1(4), 7-32. 
Nijenhuis, E. R. S. (2010). THE SCORING AND INTERPRETATION OF THE SDQ-20 AND SDQ-5. Activitas Nervosa Superior, 52(1), 24-28.

Nijenhuis, E. R. S., Spinhoven, P., Van Dyck, R., van der Hart, O., \& Vanderlinden, J. (1996). The development and psychometric characteristics of the Somatoform Dissociation Questionnaire (SDQ-20). The Journal of Nervous and Mental Disease, 184(11), 688-694.

Nimnuan, C., Hotopf, M., \& Wessely, S. (2001). Medically unexplained symptoms: an epidemiological study in seven specialities. Journal of Psychosomatic Research, 51(1), $361-7$.

Preston, C., \& Newport, R. (2011). Analgesic effects of multisensory illusions in osteoarthritis. Rheumatology (Oxford, England), 50(12), 2314-5. doi:10.1093/rheumatology/ker104

Proske, U., \& Gandevia, S. C. (2012). The proprioceptive senses: their roles in signaling body shape, body position and movement, and muscle force. Physiological Reviews, 92(4), 1651-97. doi:10.1152/physrev.00048.2011

Reid, S., Wessely, S., Crayford, T., \& Hotopf, M. (2001). Medically unexplained symptoms in frequent attenders of secondary health care: retrospective cohort study. BMJ (Clinical Research Ed.), 322(7289), 767.

Rief, W., \& Barsky, A. J. (2005). Psychobiological perspectives on somatoform disorders. Psychoneuroendocrinology, 30(10), 996-1002. doi:10.1016/j.psyneuen.2005.03.018

Sar, V., Kundakci, T., Kiziltan, E., Bakin, B., \& Bozkurt, O. (2001). Differentiating Dissociative Disorders from Other Diagnostic Groups Through Somatoform Dissociation in Turkey. Journal of Trauma \& Dissociation, 1(4), 67-80. doi:10.1300/J229v01n04

Schaefer, M., Egloff, B., \& Witthöft, M. (2012). Is interoceptive awareness really altered in 
somatoform disorders? Testing competing theories with two paradigms of heartbeat perception. Journal of Abnormal Psychology, 121(3), 719-24. doi:10.1037/a0028509

Scholz, O. B., Ott, R., \& Sarnoch, H. (2001). Proprioception in somatoform disorders. Behaviour Research and Therapy, 39(12), 1429-38.

Sierra, M., \& David, A. S. (2011). Depersonalization: A selective impairment of selfawareness. Consciousness and Cognition, 20(1), 99-108. doi:10.1016/j.concog.2010.10.018

Singmann, H., Bolker, B., \& Westfall, J. (2015). afex: Analysis of Factorial Experiments.

Spielberger, C. D., Gorsuch, R. L., Lushene, P. R., Vagg, P. R., \& Jacobs, A. G. (1983). Manual for the State-Trait Anxiety Inventory (Form Y). Manual for the statetrait anxiety inventory STAI.

Steinbrecher, N., Koerber, S., Frieser, D., \& Hiller, W. (2011). The prevalence of medically unexplained symptoms in primary care. Psychosomatics, 52(3), 263-71. doi:10.1016/j.psym.2011.01.007

Welch, R. B., \& Warren, D. H. (1980). Immediate perceptual response to intersensory discrepancy. Psychological Bulletin, 88(3), 638-667. doi:10.1037//0033-2909.88.3.638

Winter, B. (2013). Linear models and linear mixed effects models in R with linguistic applications. Retrieved from arXiv:1308.5499 
Table 1. Descriptive statistics for age (years), SDQ scores, SSAS and STAI-T scores for participants split by SDQ group.

\begin{tabular}{|c|c|c|c|c|c|}
\hline & SDQ group & Minimum & Maximum & Mean & Standard deviation \\
\hline \multirow[t]{2}{*}{ Age } & Low & 18.61 & 45.04 & 27.67 & 8.68 \\
\hline & High & 19.59 & 29.12 & 23.35 & 2.83 \\
\hline \multirow[t]{2}{*}{ SDQ score } & Low & 20 & 21 & 20.24 & .44 \\
\hline & High & 26 & 39 & 28.93 & 3.45 \\
\hline \multirow[t]{2}{*}{ SSAS score } & Low & 16 & 42 & 27.71 & 7.44 \\
\hline & High & 26 & 38 & 32.15 & 3.93 \\
\hline STAI-T & Low & 22 & 60 & 37.94 & 10.02 \\
\hline score & High & 31 & 68 & 39.36 & 9.43 \\
\hline
\end{tabular}


Table 2: Mean (SD) localisation error values $(\mathrm{cm})$ in the congruent conditions

\begin{tabular}{rrrcc}
\hline \multicolumn{1}{l}{ Hand } & SDQ group & CS & CUI & CUO \\
\hline Left & Low & $-.04(.19)$ & $.51(1.24)$ & $.73(1.40)$ \\
& High & $-.05(.20)$ & $.60(1.43)$ & $-.28(1.20)$ \\
Right & Low & $-.01(.21)$ & $.35(1.18)$ & $.05(1.87)$ \\
& High & $-.02(.24)$ &. $.12(1.53)$ & $-.10(1.94)$ \\
\hline
\end{tabular}




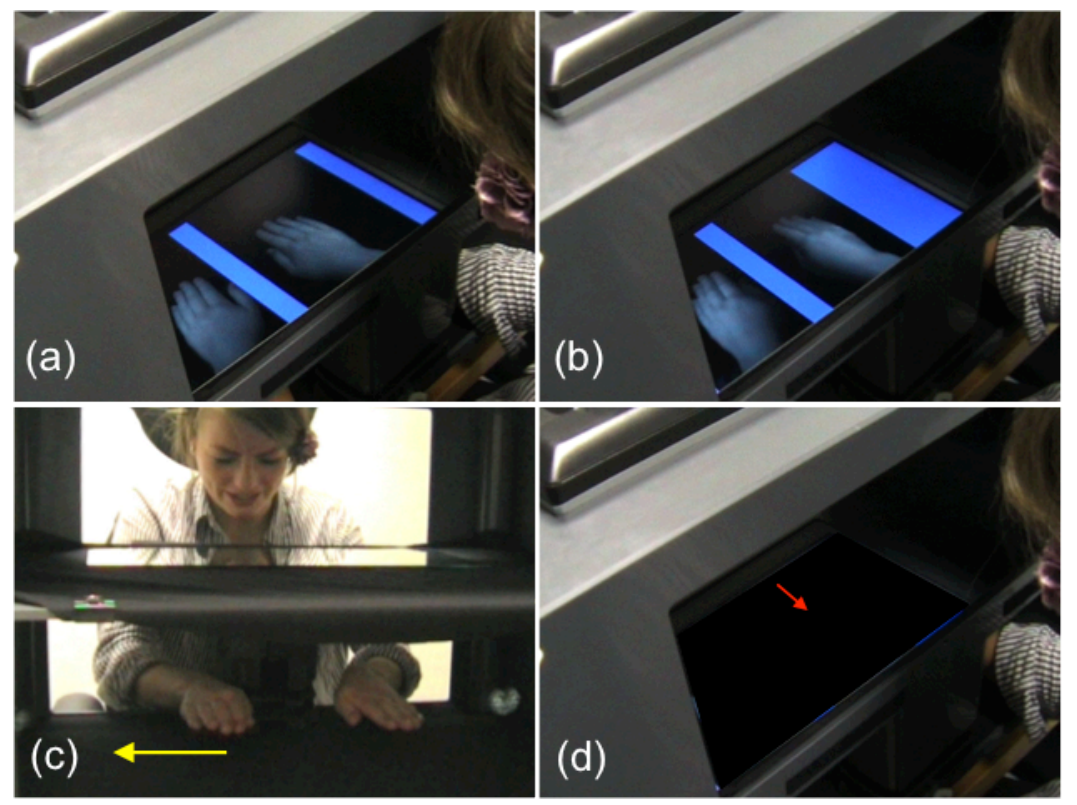

Figure 1. (a) The adaptation procedure began with the seen and real locations of the right hand in alignment; (b) Superimposed blue bars slowly expanded to constrict the space around the hand over the course of the adaptation procedure while, at the same time and without the participant's awareness, the image of the right hand moved slowly leftwards so that in order to keep the hand visible between the blue bars, the participant had to move their real hand rightwards creating a discrepancy between the seen and real location of the hand - note the misalignment of the seen right hand and the participant's real arm compared to (a). A bib occluded the participant's view of their arms during the actual experiment. (c) The participant's hands on the MIRAGE work surface from the experimenter's viewpoint. The yellow arrow indicates the direction of movement for the right hand during adaptation. (d) Following occlusion of the hand and work surface the participant indicated the felt location of their real hand by saying "Stop" when a moving arrow was in line with where they felt their index finger to be. 


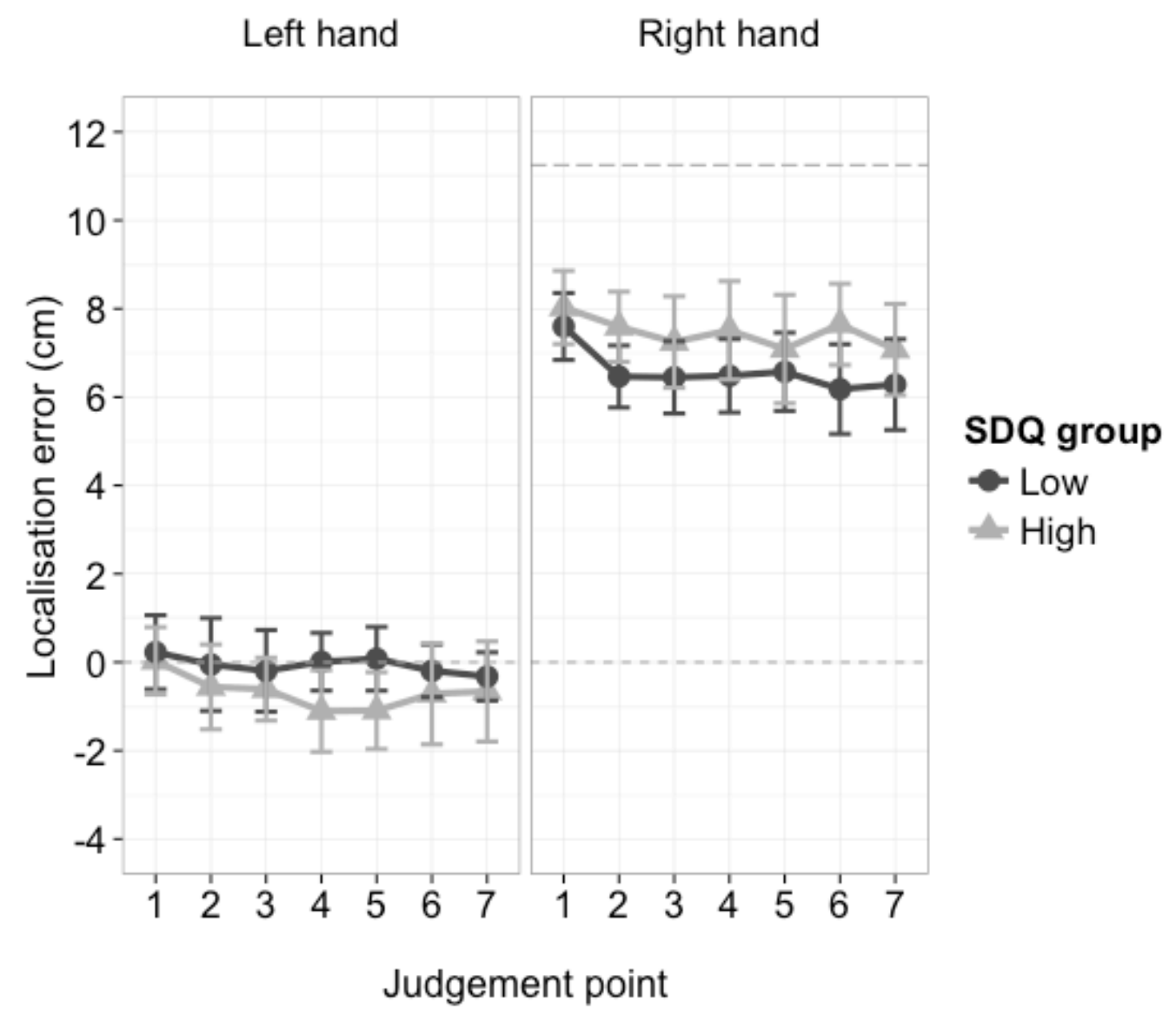

Figure 2. Mean localisation error in the incongruent condition at each judgement point (seven across an 8 minute period) for each SDQ group split by hand. Dashed line at $y=0$ indicates actual location of the hand, dashed line at $y=11.25$ indicates seen location of the right hand (left hand seen at actual location). Error bars $=95 \%$ CI. 